\title{
Kommentar II zum Fall: „Palliativmedizin im interkulturellen Kontext"“
}

\author{
Silke Schicktanz
}

Online publiziert: 22. Januar 2010

(C) Springer-Verlag 2010

Der geschilderte Fall ist paradigmatisch dafür, dass die Rücksicht auf kulturelle Bedürfnisse von Patienten und Angehörigen am Lebensende sehr aufwendig und komplex sein kann. Jedoch stellen sich auch unabhängig vom türkisch-muslimischen Hintergrund des Patienten und seiner Familie zwei zentrale ethische Probleme:

- Indikation und moralische Rechtfertigung einer terminalen Sedierung

- Die Rolle von Familienangehörigen bei Entscheidungen am Lebensende

Durch den Religions- und Migrationshintergrund kommen zwei weitere wichtige Fragen hinzu:

- Welche Rolle können und sollen religiöse Autoritäten bei konkreten medizinethischen Entscheidungen spielen?

- Wie geht man mit Verständigungsproblemen zwischen Patienten/Angehörigen und Ärzteschaft um, wenn Information, Aufklärung und Willensäußerung zentral für die ethische Legitimation von Entscheidungen sind?

Die durchgeführte terminale (tief-kontinuierliche) Sedierung im Fall des ALS-Patienten im sehr fortgeschrittenen Krankheitsstadium ist eine nachvollziehbare Therapie, wenn - wie geschildert - es sich erstens um eine extrem schwere Leidenssituation (massive Atemnot, Angstzustände) einer unheilbaren Erkrankung im Endstadium handelt und zweitens der ausdrückliche Wille des Patienten hierfür vorliegt. Beides war auf den ersten Blick gegeben, da der Patient noch selbst kommunizierte (wenngleich eingeschränkt und vermittelt über Dritte). Bezüglich der Entscheidungsfähigkeit kann eingewendet werden, dass durch die erste palliative Versorgung mit Midazolam und Morphin eine Einschränkung der Bewusstseins- und Entscheidungsfähigkeit nicht auszuschließen war. Die reduzierte Form der Kommunikation (über Augenblinzeln und in türkischer Sprache) hat vermutlich eine Sicherstellung der Entscheidungsfähigkeit durch das ärztliche Team erschwert (ob dies überhaupt

S. Schicktanz $(\bowtie)$

Abteilung Ethik und Geschichte der Medizin, Universitätsmedizin Göttingen

Humboldtallee 36, 37073 Göttingen, Deutschland

E-Mail: silke.schicktanz@medizin.uni-goettingen.de 
erwogen wurde, wird nicht erläutert). Der längere Entscheidungsprozess samt Hinzuziehen einer religiösen Autorität zeigt allerdings an, dass dem Patienten die moralische Dimension dieser medizinischen Behandlung durchaus bewusst war, sonst hätte er nicht unbedingt solch einen Rat eingeholt.

Mir scheint aus ethischer Sicht in diesem Falle zentral, dass die Ärzte dem Imam und dem Patienten die Einschätzung gegeben haben, dass die tief-kontinuierliche Sedierung in diesem Falle keine Lebenszeitverkürzung darstellt. Dies war für den religiösen Patienten ganz wesentlich für seine Entscheidung. Man muss allerdings fragen, warum dem Patienten eine maschinelle Unterstützung der Beatmung nicht angeboten wurde. Dieses Vorgehen ist bei ALS-Patienten in diesem Stadium nicht unüblich. Wenn dem so wäre und keine medizinische Kontraindikation dafür vorlag, so fragt man sich, ob dann die Interpretation nicht anders ausfallen müsste, d.h. es sich im Vergleich zu anderen ALS-Patienten doch um eine Maßnahme handeln würde, bei der eine Lebenszeitverkürzung (wenngleich nicht intendiert, sondern als Nebenwirkung) in Kauf genommen werden müsste. Dies stellt ja für viele Patienten auch kein moralisches Problem dar. Wenn also die Eindeutigkeit der Interpretation, wie hier suggeriert, gar nicht gegeben war, so muss die Informationsgabe an Patient und Imam problematisiert werden. Bei Unsicherheiten oder entgegengesetzter medizinischer Einschätzung hätte sich der Imam sehr wahrscheinlich gegen die terminale Sedierung ausgesprochen [2]. Wie wichtig dem Patienten die Vermeidung der Lebenszeitsverkürzung war, zeigt auch die gewünschte Sondenernährung und Flüssigkeitszufuhr. Auch wenn das Hinzuziehen von religiösen Autoritäten als Ausdruck von Patientenselbstbestimmung an sich sehr zu begrüßen ist, macht der Fall deutlich, dass die moralisch-theologische Empfehlung enorm von Information, Hintergrundwissen und Problemkompetenz der jeweiligen religiösen Autorität abhängt. Dies ist nicht unproblematisch. Rücksicht auf kulturelle Eingebettetheit darf nicht bedeuten, intrakulturelle Widersprüche und Konflikte (z.B. verschiedene Islamschulen beurteilen medizinethische Probleme unterschiedlich) [2] zu verharmlosen.

Die Einbeziehung von Angehörigen bei Entscheidungen am Lebensende ist nicht nur wünschenswert, sondern dringend geboten. Es ist davon auszugehen, dass Angehörige fast immer psychosozialer Betreuung im Trauer- und Betreuungsprozess bedürfen. Aus diesem Grunde würde ich darin eine Aufgabe der medizinisch-palliativen Versorgung sehen [1]. Die Einbeziehung von Angehörigen (insbesondere bei Entscheidungsprozessen) bedarf jedoch auch der höheren Sensibilität für potentielle intrafamiliäre Konflikte. Dort, wo Patienten und Angehörige sich einig sind, ist die Einbeziehung gerade nicht prekär. Im geschilderten Fall ist davon auszugehen, dass der Patient durchaus gegen den Willen seiner Eltern handelte, auch wenn der Imam es erlaubte (aber nicht gebot). Der Vater hat in dem genannten Fall nicht nur die eingeleitete Sedierung abbrechen lassen, sondern letztlich das „Wie“ beeinflusst.

Das Aufbrechen von unterschwelligen Konflikten und Kritik an hierarchischen, traditionellen Familienstrukturen ist in existenziellen Situationen eher wahrscheinlich als im funktionierenden Alltag. Umso mehr bedarf es also der „Vorsorge“ solcher Konflikte. Stellt man die Patientenautonomie in den Vordergrund, so hieße das, auch auf die Wahrscheinlichkeit von intrafamiliären Konflikten in derartigen Situationen hinzuweisen. Möglicherweise hätte im Vorfeld geklärt werden können, ob der Sohn seinen Vater als Bevollmächtigten für die eintretende Phase der Entscheidungsunfähigkeit aktiv benennt. Damit wäre der Wille des Vaters direkt autorisiert gewesen. Es ist bekannt, dass viele Angehörige während der terminalen Sedierung genau darunter leiden, dass der Kommunikationsprozess abbricht [1]. Daher ist die Reaktion des Vaters sehr nachvollziehbar. In dem vorliegenden Fall hat sie zu einer eher kurzen Verlängerung des Prozesses (nur um zwei Stunden) geführt. Aber hier 
deutet sich ein grundsätzliches Problem an, wenn Angehörige nach möglichem irreversiblen Verlust der Kommunikations- und Entscheidungsfähigkeit die vorher getroffene Entscheidung des Patienten dann in Frage stellen und ggf. die Umsetzung der vorher abgesprochenen Entscheidung aufhalten.

\section{Literatur}

1. Neitzke G et al. (2009) Sedierung am Lebensende: Eckpunkte für einen verantwortungsvollen Umgang. In: Vollmann J, Schildmann J, Simon A (Hrsg) Klinische Ethik. Campus, Frankfurt a. M., S 185-207

2. Sachedina A (2005) End-of-life: the Islamic view. Lancet 366:774-779 